\title{
Asymptotic properties of the hazard function and the mean residual life function
}

\author{
DEJAN ŠKANATA \\ Enconet d.o.o., Zagreb \\ University of Applied Sciences Velika Gorica, Velika Gorica \\ Faculty of Electrical Engineering and Computing, University of Zagreb
}

CROATIA

\begin{abstract}
The memoryless or non-aging property of systems is of special relevance in reliability theory, which implies that the hazard function is constant in time, and the corresponding mean residual life function takes a reciprocal value. The only known continuous distribution with that property is the exponential distribution. However, many other distributions exist whose asymptotic behavior of underlying hazard functions approaches a constant, while the mean residual life function approaches a reciprocally constant value. Here we provide an analysis which enables us to study a class of distributions that asymptotically approach the memoryless property, and which include gamma, Erlangian, exponential resilience, exponential geometric, hyper exponential, logistic exponential and the inverse Gaussian distribution.
\end{abstract}

Key-Words: hazard function, mean residual life function, memoryless property

Received: November 16, 2019. Revised: May 7, 2020. Re-revised: May 29, 2020. Accepted: May 31, 2020. Published: June 2, 2020.

\section{Introduction}

Modeling lifetime data usually involves the hazard function in conjunction with other lifetime measures, particulraly the mean residual life function. The hazard function, $h(t)$, defined at a point in time $t$ provides information about operation of an item in a short time interval immediately after $t$. The mean residual life function, $m(t)$, at a point in time $t$ considers information which spread over the complete future operation of an item. This intuition explains difference between two functions.
Both of them are conditional functions and are needed in reliability theory and its practical applications.

The main properties of $h(t)$ and $m(t)$, which include their probabilistic interpretation, relationship with the reliability function, mutual relationship, mathematical properties and descriptive shapes are given in Table 1. Information shown in the table is mainly based on Leemis [1], Singpurwalla [2], Lai and Xie [3], Finkelstein [4], Rinne [5] and O'Connor et al. [6].

Table 1 The main properties of the hazard function and the mean residual life function

\begin{tabular}{|c|c|c|}
\hline Property & $h(t)$ & $m(t)$ \\
\hline \multirow{2}{*}{$\begin{array}{l}\text { Probabilistic } \\
\text { interpretation }\end{array}$} & $\begin{array}{l}\text { Approximately equal to the probability of failure } \\
\text { in a small unit of time } d t \text {, given that no failure } \\
\text { occurred up to time } t \text {. }\end{array}$ & $\begin{array}{l}\text { Expected remaining life of an item that has been } \\
\text { operating without failure up to time } t \text {. }\end{array}$ \\
\hline & $h(t) d t \approx \operatorname{Pr}(t \leq T \leq t+d t \mid T \geq t)$ & $m(t)=E\left[\frac{T-t}{T>t}\right]$ \\
\hline $\begin{array}{l}\text { Relationship with the } \\
\text { reliability function }\end{array}$ & $h(t)=\frac{f(t)}{R(t)}$ & $m(t)=\frac{\int_{t}^{\infty} R(\tau) d \tau}{R(t)}$ \\
\hline Mutual relationship & \multicolumn{2}{|c|}{$h(t)=\frac{1+m^{\prime}(t)}{m(t)}$} \\
\hline \multirow[t]{2}{*}{$\begin{array}{l}\text { Mathematical } \\
\text { properties }\end{array}$} & $\begin{array}{ll}\text { 1. } & h(t) \geq 0 \\
\text { 2. } & \int_{0}^{\infty} h(t) d t \rightarrow \infty\end{array}$ & $\begin{array}{ll}\text { 1. } & m(t) \geq 0 \\
\text { 2. } & m(0)=\mu \\
\text { 3. } & m^{\prime}(t) \geq-1 \\
\text { 4. } & \int_{0}^{\infty} \frac{1}{m(t)} d t \rightarrow \infty\end{array}$ \\
\hline & $\begin{array}{l}\text { Right continuous. Constant (CFR), increasing } \\
\text { (IFR), decreasing (DFR), bathtub curve (BTFR), } \\
\text { up-side-down bathtub curve (UBTFR), roller- } \\
\text { coaster curve and different oscillatory curves. }\end{array}$ & $\begin{array}{l}\text { Right continuous. Constant (CMRL), increasing } \\
\text { (IMRL), decreasing (DMRL), bathtub curve } \\
\text { (BTMRL) and up-side-down bathtub curve } \\
\text { (UBTMRL). }\end{array}$ \\
\hline
\end{tabular}


Note that Table 1 uses following abbreviations:

- Hazard function (usually abbreviated as HF) is a term used across different scientific disciplines. In reliability theory this function is commonly known as the failure rate and is abbreviated as FR which is used in the table.

- MRL is abbreviation commonly used for the mean residual (remaining) life function.

\section{Conditional reliability and memoryless property}

Conditional reliability of an item at age $t_{o}$ is defined with:

$R\left(t \mid t_{o}\right)=\frac{R\left(t_{o}+t\right)}{R\left(t_{o}\right)}$

If $R\left(t \mid t_{0}\right)$ equals $R(t)$, the item at age $t_{o}$ has the same reliability characteristic as it had before $t_{o}$. In other words, the operation history of the item is irrelevant to its future operation. This is known as the memoryless or non-aging property. Important consequence of this property is that $h(t)$ is constant in time and $m(t)=1 / h(t)$.

It is well-known that the memoryless property is satisfied only by the exponential distribution in the continuous domain. For this distribution the following is valid:

$f(t)=\lambda e^{-\lambda t}$

$R\left(t \mid t_{o}\right)=\frac{e^{-\lambda\left(t_{o}+t\right)}}{e^{-\lambda t_{o}}}=e^{-\lambda t}=R(t)$

$h(t)=\frac{f(t)}{R(t)}=\lambda$

$m(t)=\frac{\int_{t}^{\infty} R(\tau) d \tau}{R(t)}=\frac{1}{\lambda}$

where $\lambda$ is the scale parameter of the exponential distribution. It should be noted that $1 / \lambda$ is the mean value of the exponential distribution. So, $m(0)$ equals the mean time of the exponential distribution.

\section{Asymptotic reciprocal relationship}

Calabria and Pulcini [7] analyzed asymptotic behavior of $m(t)$ and $h(t)$ functions. By applying the L'Hospital rule they derived the following asymptotic reciprocal relationship between them:

$$
\begin{aligned}
& \lim _{t \rightarrow \infty} m(t)=\lim _{t \rightarrow \infty} \frac{\int_{t}^{\infty} R(\tau) d \tau}{R(t)} \lambda e^{-\lambda t}= \\
& =\lim _{t \rightarrow \infty} \frac{-R(t)}{\frac{d R(t)}{d t}}=\lim _{t \rightarrow \infty} \frac{R(t)}{f(t)}=\lim _{t \rightarrow \infty} \frac{1}{h(t)}
\end{aligned}
$$

Calabria and Pulcini claim that this relationship is in agreement with intuition. Namely, as $t \rightarrow \infty$, the immediate operation of an item tends to coincide with its complete future operation.

Bradley and Gupta [8] devoted a part of their paper to the Calabria and Pulcini asymptotic reciprocal relationship by showing that

$\lim _{t \rightarrow \infty}[m(t) h(t)]=1$

is not true in general. They provided a simple counterexample by considering the class of distributions with linear mean residual life function. However, they stated that the reciprocal asymptotic relationship may be interpreted as an approximation.

In many cases is not easy to determine $m(t)$. In such cases Calabria and Pulcini used the following useful relationship between the asymptotic behavior of $m(t)$ and the probability density function, $f(t)$ :

$\lim _{t \rightarrow \infty} m(t)=\lim _{t \rightarrow \infty}-\frac{1}{\frac{d}{d t} \ln [f(t)]}$

which is also given in Lawless [9]. But Calabria and Pulcini failed to notice explicitly that their and Lawless relationship are equivalent. Namely, by appropriate substitution of $h(t)$ in Calabria and Pulcini relationship and by applying the L'Hospital rule the following is true:

$$
\begin{aligned}
& \lim _{t \rightarrow \infty} \frac{1}{h(t)}=\lim _{t \rightarrow \infty} \frac{\int_{t}^{\infty} f(\tau) d \tau}{f(t)}=\lim _{t \rightarrow \infty} \frac{-f(t)}{\frac{d f(t)}{d t}}= \\
& =\lim _{t \rightarrow \infty}-\frac{1}{\frac{d}{d t} \ln [f(t)]}
\end{aligned}
$$

By applying the similar procedure, we can derive asymptotic relationships between $m(t)$ and other popular lifetime representations such as the reliability function, the cumulative function and the cumulative hazard function.

Calabria and Pulcini made calculations of $h(t)$ and $m(t)$ asymptotic values for some univariate continuous distributions such as the exponential, Weibull, gamma, normal, lognormal and the inverse Gaussian distribution. According to their results the

\begin{tabular}{|c|c|c|}
\hline Distribution class & $\lim _{t \rightarrow \infty} h(t)$ & $\lim _{t \rightarrow \infty} m(t)$ \\
\hline $\begin{array}{l}\text { Exponential, gamma } \\
\text { and inverse Gaussian }\end{array}$ & Constant & $\begin{array}{c}\text { Reciprocally } \\
\text { constant }\end{array}$ \\
\hline $\begin{array}{l}\text { Weibull }(\beta<1) \text { and } \\
\text { lognormal }\end{array}$ & 0 & $\infty$ \\
\hline $\begin{array}{l}\text { Weibull }(\beta>1) \text {, } \\
\text { normal and distributions } \\
\text { defined in finite interval }\end{array}$ & $\infty$ & 0 \\
\hline
\end{tabular}
following three classes of distributions may be recognized (Table 2 ).

Table 2 Distribution classes based on [7] 
For distributions defined on finite interval $[a, b]$ the following is valid:

$\lim _{t \rightarrow b} h(t)=\infty$

$m(b)=0$

\section{Approaching memoryless property}

In this work we analyze the asymptotic behavior of several univariate continuous distributions. Particular attention is paid to distributions that belong to the distribution class for which the asymptotic reciprocal relationship between $h(t)$ and $m(t)$ holds. To compute the asymptotic form of $m(t)$ we use (6) where that is possible, and (8) otherwise. In addition to distributions mentioned in Table 2 we find also the Erlangian, exponential geometric, exponential resilience, hyper exponential and the logistic exponential distribution follow this rule. Mathematical form of these distributions follows Lai and Xie [3], Finkelstein [4], O'Conor et al. [6], and Leemis [10]. The results are shown in Table 3.

Table 3 Asymptotic behavior of the univariate continuous distributions analyzed in the paper

\begin{tabular}{|c|c|c|c|c|c|c|c|c|}
\hline \multirow[b]{2}{*}{ Distribution $^{1)}$} & \multirow[b]{2}{*}{$\begin{array}{c}\text { Shape } \\
\text { parameter }\end{array}$} & \multicolumn{3}{|c|}{$h(t)$} & \multicolumn{3}{|c|}{$m(t)$} & \multirow[b]{2}{*}{$\lim _{t_{o} \rightarrow \infty} R\left(t \mid t_{o}\right)^{7)}$} \\
\hline & & $\boldsymbol{h}(\mathbf{0})$ & $\lim _{t \rightarrow \infty} h(t)$ & Shape & $m(0)^{2)}$ & $\lim _{t \rightarrow \infty} m(t)$ & Shape & \\
\hline Exponential & - & $\lambda$ & $\lambda$ & CFR & $\frac{1}{\lambda}$ & $\frac{1}{\lambda}$ & CMRL & $e^{-\lambda t}$ \\
\hline \multirow{2}{*}{ Gamma } & $\alpha>1$ & 0 & \multirow{2}{*}{$\lambda$} & IFR & \multirow{2}{*}{$\frac{\alpha}{\lambda}$} & \multirow{2}{*}{$\frac{1}{\lambda}$} & DMRL & \multirow{2}{*}{$e^{-\lambda t}$} \\
\hline & $\alpha<1$ & $\infty$ & & DFR & & & IMRL & \\
\hline Erlangian & $n>1$ & 0 & $\lambda$ & IFR & $\frac{n}{\lambda}$ & $\frac{1}{\lambda}$ & DMRL & $e^{-\lambda t}$ \\
\hline $\begin{array}{l}\text { Exponential } \\
\text { resilience }^{3)}\end{array}$ & $\alpha>1$ & 0 & $\lambda$ & IFR & $\frac{H N(\alpha)}{\lambda}$ & $\frac{1}{\lambda}$ & DMRL & $e^{-\lambda t}$ \\
\hline \multirow{2}{*}{$\begin{array}{l}\text { Exponential } \\
\text { geometric }\end{array}$} & $\beta>1$ & \multirow{2}{*}{$\frac{\lambda}{\beta}$} & \multirow{2}{*}{$\lambda$} & IFR & $\beta \ln (\beta)$ & \multirow{2}{*}{$\frac{1}{\lambda}$} & DMRL & \multirow{2}{*}{$e^{-\lambda t}$} \\
\hline & $\beta<1$ & & & DFR & $\overline{\lambda(\beta-1)}$ & & IMRL & \\
\hline $\begin{array}{l}\text { Hyper } \\
\left.\text { exponential }^{4}\right)\end{array}$ & $\sum_{i} \alpha_{i}=1$ & $\sum_{i} \alpha_{i} \lambda_{i}$ & $\lambda$ & DFR & $\sum_{i} \frac{\alpha_{i}}{\lambda_{i}}$ & $\frac{1}{\lambda}$ & IMRL & $e^{-\lambda t}$ \\
\hline \multirow{2}{*}{$\begin{array}{l}\text { Logistic } \\
\text { exponential }^{5 \text { ) }}\end{array}$} & $\alpha>1$ & 0 & \multirow{2}{*}{$\lambda \alpha$} & UBTFR & \multirow{2}{*}{-} & \multirow{2}{*}{$\frac{1}{\lambda \alpha}$} & BTMRL & \multirow{2}{*}{$e^{-\lambda \alpha t}$} \\
\hline & $\alpha<1$ & $\infty$ & & BTFR & & & UBTMRL & \\
\hline $\begin{array}{l}\text { Inverse } \\
\text { Gaussian }\end{array}$ & $\mu>0$ & 0 & $\frac{\lambda}{2 \mu^{2}}$ & UBTFR & $\mu$ & $\frac{2 \mu^{2}}{\lambda}$ & BTMRL & $e^{-\frac{\lambda}{2 \mu^{2}} t}$ \\
\hline
\end{tabular}

The footnotes in Table 3 are:

1) For all distributions listed the scale parameter is $\lambda$.

2) $m(0)=\mu$ means that value of the $m(t)$ function at $t=0$ equals the mean value of the supporting distribution.

3) Mathematical form is taken from Finkelstein [4]. This distribution has not received much attention in the reliability literature. $H N(\alpha)$ is abbreviation for the Harmonic Number function.

4) Mathematical form is taken from Lai and Xie [3]. A two-component distribution is considered with $\alpha_{1}+\alpha_{2}=1$ and $\lambda_{1}=\lambda_{2}=\lambda$.

5) Mean value as well as $m(0)$ is not found analytically. Median equals $\ln (2) / \lambda$.

6) $\mu$ is the mean value of the inverse Gaussian distribution.
7) The following is true:

$$
\begin{aligned}
& \lim _{t_{o} \rightarrow \infty} R\left(t \mid t_{o}\right)=e^{-\lim _{t_{o \rightarrow \infty}} h(t)}= \\
& =e^{-\lim _{t_{o} \rightarrow \infty} \frac{1}{m(t)}}
\end{aligned}
$$

The common characteristics of distributions mentioned above are as follows:

- When the shape parameter equals 1, all distributions tend towards the exponential distribution. Exception is the inverse Gaussian distribution because it is characterized by mean value not the shape parameter.

- As $t_{o} \rightarrow \infty$ the conditional reliability for distributions tends to exponential reliability, meaning that they are approaching memoryless property. 
- The closer shape parameter is to 1 , the memoryless approaching process is faster. This is not valid for the inverse Gaussian distribution.

It should be noted here that the author's most recent research on this topic shows that the chisquared distribution and the extreme value distribution (also known as Gumbel maximum) as well as the Laplace distribution, in certain circumstances exhibit very similar properties as the distributions listed in Table 3.

As an example of the performed calculations Fig.1 shows $h(t)$ and $m(t)$ functions of the Erlangian distribution which is a special case of the gamma distribution. This distribution plays an important role in reliability engineering. For example, it is the distribution function of the time to failure of a cold standby system where the lifetimes of components are exponentially distributed.

The Erlangian distribution is two-parameter distribution with $\lambda$ as the scale parameter of the exponential distribution while $n$ is a positive integer. For this distribution the following is true:

$f(t)=\frac{\lambda(\lambda t)^{n-1}}{(n-1) !} e^{-\lambda}$

$R(t)=\frac{\Gamma(n, \lambda t)}{\Gamma(n)}$

$h(t)=\frac{(\lambda t)^{n}}{t \Gamma(n, \lambda t)} e^{-\lambda t}$

$m(t)=\frac{\Gamma(1+n, \lambda t)}{\lambda \Gamma(n, \lambda t)}-t$

$\Gamma(n)$ in the above formulas is gamma function while $\Gamma(n, \lambda t)$ is incomplete gamma function. To determine $m(t)$ we use the following formula from Finkelstein [4]:

$m(t)=\frac{\int_{t}^{\infty} \tau f(\tau) d \tau}{R(t)}-t$

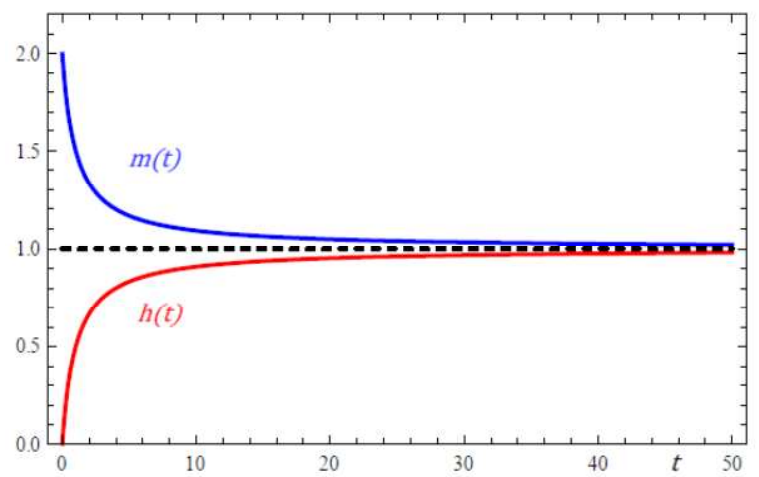

Fig. $1 h(t)$ (red) and $m(t)$ (blue) of the Erlangian distribution with $\lambda=1$ and $n=2$

It is quite clear from Fig. 1 that as $t \rightarrow \infty, h(t)$ increases towards $\lambda$, while $m(t)$ decreases towards
$1 / \lambda$, showing that the Erlangian distribution asymptotically approaches memoryless property.

\section{A short note on the oscillatory hazard function}

As mentioned in Table 1, the hazard function may take different shapes. Among others it may take a shape of different oscillatory curves. This has not received any attention in the reliability literature so far. Let us analyze the following hazard function:

$h(t)=\lambda+\frac{\sin \pi\left(t-t_{1}\right)}{\pi\left(t-t_{1}\right)}$

which represents attenuated oscillations around parameter $\lambda$ with maximum amplitude (maximum system disturbance) of $\lambda+1$ at time $t_{1}$. The corresponding probability density function (or supporting distribution) and the reliability function are:

$f(t)=\left[\lambda+\frac{\sin \pi\left(t-t_{1}\right)}{\pi\left(t-t_{1}\right)}\right] e^{-t-\frac{S I\left(\pi t_{1}\right)-S I\left[\pi\left(t_{1}-t\right)\right]}{\pi}}$

$R(t)=e^{-t-\frac{S I\left(\pi t_{1}\right)-S I\left[\pi\left(t_{1}-t\right)\right]}{\pi}}$

where $S I$ denotes Sine integral function as described by Weisstein [11]. In this particular example the following may be shown:

$\lim _{t \rightarrow \infty} h(t)=\lambda$

$\lim _{t \rightarrow \infty} m(t)=\lim _{t \rightarrow \infty}-\frac{1}{\frac{d}{d t} \ln [f(t)]}=\frac{1}{\lambda}$

$\lim _{t_{o} \rightarrow \infty} R\left(t \mid t_{o}\right)=e^{-\lambda t}$

Therefore, as $t_{o} \rightarrow \infty$ the distribution supporting attenuated oscillations in $h(t)$, asymptotically approaches memoryless property.

The same results apply to the following attenuated oscillatory hazard functions:

$h(t)=S I(t)$

$h(t)=$ FresnelC $(t)$

$h(t)=$ Fresnel $(t)$

\section{Conclusions}

In this paper we argue:

- Both the hazard function, $h(t)$ and the mean residual life function, $m(t)$, are important lifetime representations. They provide information on short and long-term future operation of an item. Determining them is of major importance in reliability theory and obtaining information on their late-time behavior 
is useful in many contexts where such functions are analytically complex.

- Asymptotic reciprocal relationship between $m(t)$ and $h(t)$ given by Calabria and Pulcini and relationship given by Lawless are equivalent.

- Important consequence of memoryless property is that $h(t)$ has fixed (constant) value in time while $m(t)$ has reciprocal value.

- There is a class of distributions for which $h(t)$ and $m(t)$ asymptotically approach constant and reciprocal values.

- Distributions that belong to this class are the exponential, gamma, Erlangian, exponential geometric, exponential resilience, hyper exponential, logistic exponential and the inverse Gaussian.

- Distributions that may be constructed on the basis of Sine integral and Fresnel functions (these distributions support the attenuated oscillatory hazard functions) also belong to the mentioned distribution class.

- All the above mentioned distributions approach memoryless property at late times.

\section{Acknowledgments}

This paper is designed as a preparation for the course on Systems Reliability at University of Applied Sciences Velika Gorica.

\section{References:}

[1] Leemis, L.M., Lifetime Distribution Identities, IEEE Transactions on Reliability, Vol. R-35, No.2, 1986, pp. 170-174

[2] Singpurwalla, N.D. (2006), The Hazard Potential: Introduction and Overview. Journal of the American Statistical Association, Vol. 101, No. 476, 2006, pp. 1705-1717

[3] Lai, C.D. and Xie, M., Stochastic Ageing and Dependence for Reliability, Springer, 2006.

[4] Finkelstein, M., Failure Rate Modelling for Reliability and Risk, Springer, 2008.

[5] Rinne, H., The Hazard Rate, Theory and Inference, With supplementary MATLABPrograms, Justus-Liebig University, 2014.

[6] O'Connor, A.N., Modarres, M. and Mosleh, A., Probability Distributions Used in Reliability Engineering, Center for Risk and Reliability, 2016.

[7] Calabria, R. and Pulcini, G. (1987), On the Asymptotic Behavior of Mean Residual Life Function, Reliability Engineering, 19, 1987, pp. $165-170$
[8] Bradley, D.M. and Gupta, R.C., Limiting behaviour of the Mean Residual Life, Ann. Inst. Statist. Math., 55(1), 2003, pp. 217-226

[9] Lawless, J.F., Statistical Models and Methods for Lifetime Data, John Wiley \& Sons, 1982.

[10] Leemis, L.M., Web Page: http://www.math.wm.edu/ leemis/chart/UDR/ UDR.html

[11] Weisstein, E.W., Sine Integral. MathWorld - A Wolfram Web Resource: http://mathworld.wolfram.com/SineIntegral.ht $\mathrm{ml}$ 